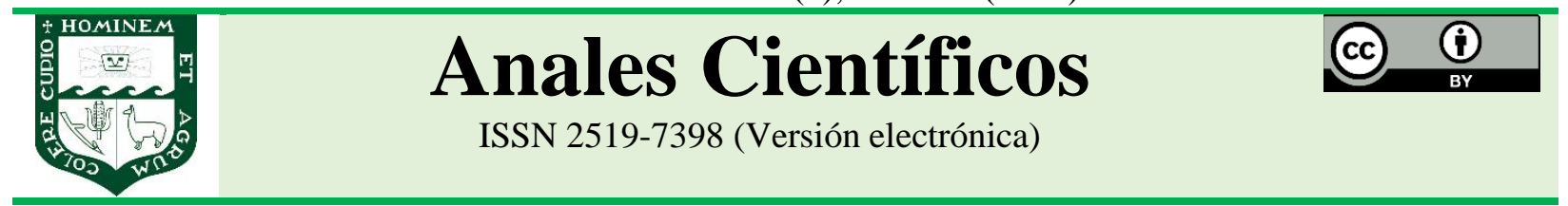

ARTÍCULO ORIGINAL - RESEARCH ARTICLE

http://dx.doi.org/10.21704/ac.v81i2.1700

\title{
EL LASALÓCIDO SÓDICO EN LA ALIMENTACIÓN DE OVINOS (OVIS ARIES) BLACKBELLY
}

\author{
Sodium lasalocid in blackbelly sheep feed (Ovis aries) \\ Jorge L. Aliaga Gutiérrez ${ }^{1 *(D)}$; Belisario A. Chian Vásquez ${ }^{1}$; José L. Cántaro Segura ${ }^{1}$ \\ ${ }^{1}$ Facultad de Zootecnia, Universidad Nacional Agraria La Molina, 15024, Lima, Perú. \\ *E-mail: jaliaga@lamolina.edu.pe
}

Recibido: 26/04/2020; Aceptado: 15/12/2020; Publicado: 30/12/2020

\begin{abstract}
The objective was to evaluate the effect of sodium lasalocid supplementation in the productive characteristics and economics in Blackbelly sheep in growth. It was used 11 male of 5 months old, randomly distributed in two groups that fed with agro-industrial residues that was added sodium alkaloid (LS) in one treatment. It was used two treatments T1 (without LS) and T2 (whit 60mg LS / kg). Feed intake $(\mathrm{kg})$, weight gain $(\mathrm{kg})$, height gain $(\mathrm{cm})$ and carcass yield (\%) was evaluated in each animal. It was used the multivariate analysis of variance of a completely randomized design with covariance for the variables feed consumption, weight gain, feed conversion and height increase. To evaluate of carcass yield it was used a completely randomized design with covariance. The results obtained in each treatment (T1 vs. T2) were: food consumption $(63,85$ vs. 63,97$)$, weight gain $(12,91$ vs. 13,33$)$, feed conversion $(4,94$ vs. 4,80$)$, size increase $(10,19$ vs. 10,09$)$ and carcass performance $(48,60$ vs. 46,66$)$. There are not statistical differences between treatments $(\mathrm{p}>0,05)$, in the variables studied. As a result of this study it is possible to conclude that the addition the $60 \mathrm{mg}$ of lasalocid sodium per $\mathrm{kg}$ in feed rations for Blackbelly sheep did not improve the performance.
\end{abstract}

Keywords: Sheep; Blackbelly; Sodium lasalocid; Supplements; Weight gain; Height increase

\section{RESUMEN}

El objetivo fue evaluar el efecto de la suplementación de lasalócido sódico en las características productivas y económicas en ovinos de raza Blackbelly en crecimiento. Se trabajó con 11 ovinos machos de 5 meses de edad, distribuidos al azar en dos grupos, que fueron alimentados con residuos agroindustriales a la cual se le adicionó lasalócido sódico (LS) según el tratamiento. Se trabajó con dos tratamientos T1 (sin LS) y T2 (con 60mg LS/ kg). Se evaluó el consumo de alimento $(\mathrm{kg})$, ganancia de peso $(\mathrm{kg})$, incremento de talla $(\mathrm{cm})$, conversión alimenticia, rendimiento de carcasa (\%), en cada animal. Se utilizó el análisis de variancia multivariado de un diseño completamente al azar con covariancia para las variables consumo de alimento, ganancia de peso, conversión alimenticia e incremento de talla. Para el rendimiento en carcasa se utilizó el diseño completamente al azar con covariancia. Los resultados obtenidos en cada tratamiento (T1 vs. T2) fueron: consumo de alimento (63,85 vs. $63,97)$, ganancia de peso $(12,91$ vs. 13,33$)$, conversión alimenticia $(4,94$ vs. 4,80$)$, incremento de talla $(10,19$ vs. $10,09)$ y rendimiento en carcasa $(48,60$ vs. 46,66$)$. No existiendo diferencias estadísticas significativas entre tratamientos $(\mathrm{p}>0,05)$, en las variables estudiadas. Los resultados de este estudio permiten concluir que la adición 
de $60 \mathrm{mg}$ de lasalócido sódico por kg de alimento en raciones de engorde para ovinos Blackbelly no mejoró la performance.

Palabras clave: Ovinos; Blackbelly; Lasalócido sódico; Suplementos; Ganancia de peso

Forma de citar el artículo (Formato APA):

Aliaga, J., Chian, B. \& Cántaro, J. (2020). El lasalócido sódico en la alimentación de ovinos (Ovis aries) Blackbelly. Anales

Científicos. 81(2), 415-423. http://dx.doi.org/10.21704/ac.v81i2.1700

Autor de correspondencia (*): Jorge L. Aliaga Gutiérrez. Email: jaliaga@lamolina.edu.pe

(C) Los autores. Publicado por la Universidad Nacional Agraria La Molina.

This is an open access article under the CC BY

\section{INTRODUCCIÓN}

La alimentación es una de las actividades más importantes y costosas en la cría de animales (Valdivia et al., 2019). En las dietas de crecimiento y finalización, el uso de aditivos alimenticios es una práctica común en los corrales de engorda (Jorquera y Angulo, 2016). Por ello, el uso de aditivos zootécnicos es una de las estrategias utilizadas para mejorar el rendimiento productivo de los animales y reducir los costos de producción (Carro et al., 2006).

La presencia de ionóforos (lasalócidos) en la dieta es necesaria para evitar el sobreconsumo y la acidosis ruminal (Pordomingo et al., 1999); además, son agentes químicos que aumentan la permeabilidad de las membranas lipídicas a los iones específicos, su acción es elevar el sodio celular y promover la salida del potasio (Michelis y Sánchez, 2016). El lasalócido sódico (LS), es una molécula biológicamente activa de un grupo conocido como ionóforo, término que está en relación a su capacidad para ayudar en el transporte de cationes a través de las membranas (Fuller y Johnson, 1981). El lasalócido sódico es un antibiótico ionóforo bivalente, producido por la fermentación de una variedad de Streptomices lasaliencis y pertenece a un grupo de compuestos naturales y sintéticos, capaces de formar cationes lipídicos (Paienter et al., 1982). El lasalócido sódico tiene un peso molecular de 612, cuya fórmula empírica es C34 H53 $08 \mathrm{Na}$, siendo soluble en agua y en la mayoría de los solventes orgánicos (Bergen y Bates, 1984). El lasalócido sódico es un polvo marrón claro fácilmente deslizable, en forma de premezcla que contiene $15 \%$ de lasalócido sódico y no es pulverulento ni higroscópico. El producto tiene una excelente estabilidad, tanto en estado puro como en alimentos molidos y peletizados, en suplementos proteicos, mezclas minerales y sal común. Los resultados de pruebas realizadas demuestran que este ionóforo se mezcla fácilmente con otros ingredientes alimenticios y pueden ser distribuidos uniformemente en la pre mezcla y en el alimento de los animales. Este producto contiene un número mínimo aproximado de partículas de 43,000 millones/gramo, densidad aproximada de $0,46 \mathrm{~kg} / \mathrm{lt}$, un tamaño promedio de partículas de 1,95 um y una gravedad específica de 1,39 g/cc (Roche, 1986).

Los efectos del lasalócido sódico sobre el rumen para modificar la fermentación se debe a la selección de un microbio comúnmente resistente al antibiótico que produce más propionato, pero menos acetato, butirato y lactato e indirectamente menos metano (Dennis et al., 1981).

Por ello, Ricke et al. (1984) mencionan que el lasalócido sódico disminuye las concentraciones de acetato e incrementa las de propionato, lo que conduce a la disminución de la relación acetato propionato (A: P). En un estudio en corderos suplementados con lasalócido sódico las pérdidas fecales de nitrógeno fueron menores, observándose una mayor retención de nitrógeno que en corderos sin lasalócido sódico, esto podría ser un reflejo del incremento de la digestibilidad del Nitrógeno (Ricke et al., 1984); de la misma manera, estudios realizados en vacunos suplementados con ionóforos se concluye que el lasalócido sódico mejora la digestibilidad del nitrógeno (Paterson et al., 1983).

En trabajos con ovinos adicionando lasalócido sódico se encontró que alteraban la absorción, retención y flujo en el tracto digestivo de varios minerales como el $\mathrm{Na}$, K, Fe y Mg (Kirk et al., 1987). La excesiva producción de ácido láctico está involucrada en la etiología y patología de la acidosis láctica en el ganado (Bartley et al., 1979). Se indica que los ionóforos inhiben el crecimiento de la pared celular de las bacterias gram 
positivas, siendo estas las mayores productoras de lactato ruminal (Chen y Wolin, 1979). Los antibióticos ionóforos tienen eficacia en la prevención de cocciodiosis, sarcocistosis y abortos causados por infección de toxoplasma.

Por otro lado, el lasalócido sódico en bajas concentraciones utilizados en el alimento fue altamente efectivo en reducir el número de oocystos encontrados en heces de ovejas y corderos infectados naturalmente (Foreyt et al., 1981b). Asimismo, Horton y Stockdale (1981), afirman que el lasalócido sódico controla la presentación natural de coccidiosis y mejora la performance en corderos alimentados en lotes y destetados precozmente. De igual manera se han reportado numerosos efectos biológicos para los ionóforos. El lasalócido sódico estimula la contracción del corazón, incrementa el flujo de la sangre y disminuye la resistencia periférica (Pressman y Fahim, 1982).

En un ensayo con carnerillos de un año de edad mantenidos en praderas y con suplementos alimenticios durante 100 días: se observó que al adicionar $50 \mathrm{mg}$ del lasalócido sódico por animal se obtuvo ganancias promedio diarias de $313 \mathrm{~g}$ vs. $279 \mathrm{~g}$ de grupos sin lasalócido sódico lo cual significó un aumento de peso mayor en 12,2 por ciento (Roche, 1988).

Foreyt et al. (1979), durante un período de 100 días en corderos en confinamiento tratados con lasalócido sódico a razón de $100 \mathrm{mg} / \mathrm{kg}$ de alimento y corderos alimentados sin lasalócido sódico, se obtuvo un promedio de ganancia de peso de $6 \mathrm{~kg}$ más por animal que los corderos no tratados y consumieron significativamente menos alimento por cada kilogramo de peso ganado. Por otro lado, la suplementación de lasalócido sódico en ovejas en gestación bajo régimen extensivo mejora el porcentaje de corderos nacidos y pesos de corderos destetados por oveja (Thomas, 1989).

\section{MATERIALES Y MÉTODOS}

El estudio se llevó a cabo en una granja particular de ovinos, ubicada en la irrigación La Esperanza Baja, sector Granados, provincia de Huaral, departamento de Lima. Se utilizaron 11 ovinos machos Blackbelly provenientes de partos dobles, de 5 meses de edad. Los ovinos fueron distribuidos al azar en dos tratamientos: cinco (5) ovinos en el grupo Testigo y seis (6) en el grupo Experimental (Tabla 1). Los animales se alojaron en corrales individuales de $1,5 \mathrm{~m} 2$ por animal, construidos con palos de eucalipto y techo de calaminas en su totalidad. Se utilizaron comederos y bebederos individuales galvanizados, balanzas de plataforma y de reloj, hipómetro para medir la talla de los ovinos en forma semanal. El estudio se realizó con dos (2) tratamientos: T1 (Ración Testigo) y T2 (Ración Experimental), las raciones se prepararon en base de residuos agroindustriales comunes de la zona, la misma ración fue proporcionados a los dos tratamientos, con la diferencia que a la Ración Experimental se adicionó 60 mg de lasalócido sódico (LS) por kg de alimento en base seca. La ración se formuló considerando los requerimientos nutricionales de corderos recomendado por el NRC (1975) utilizando el Programa Mixit-2 al mínimo costo (Tabla 2). El análisis proximal de alimento se realizó en el Laboratorio de Análisis de Alimentos del Departamento de Nutrición de la Universidad Nacional Agraria La Molina.

Tabla 1. Características de los ovinos en los grupos experimentales en la fase inicial.

\begin{tabular}{cccc}
\hline Tratamiento & $\begin{array}{c}\text { Ovinos } \\
\mathbf{N}^{\circ} \text { Arete }\end{array}$ & $\begin{array}{r}\text { Peso } \\
(\mathbf{k g})\end{array}$ & $\begin{array}{c}\text { Talla* } \\
(\mathbf{c m})\end{array}$ \\
\hline & 1 & 20,0 & 49,0 \\
Testigo & 2 & 21,5 & 53,5 \\
(T1) & 3 & 18,5 & 55,0 \\
& 4 & 24,0 & 52,0 \\
& 5 & 19,2 & 51,5 \\
\hline Promedio & & 20,64 & 52,40 \\
D. S. & & 2,154 & 2,152 \\
\hline & 6 & 22,5 & 55,5 \\
& 7 & 19,5 & 51,5 \\
Experimental (T2) & 8 & 22,5 & 51,5 \\
& 9 & 22,0 & 53,5 \\
& 10 & 19,5 & 48,0 \\
& 11 & 24,0 & 56,5 \\
\hline Promedio & & 21,67 & 52,75 \\
D. S. & & 1,807 & 3,094 \\
\hline
\end{tabular}

* Altura a la cruz.

Antes del inicio del experimento, los animales tuvieron un periodo de adaptación de 21 días para acostumbrarse a los corrales individuales y al cambio de alimento debido a que los animales procedían de una crianza al pastoreo. Durante este periodo los animales fueron desparasitados con Ivermectina al $1 \%$ y se tomaron 
muestras de heces de todos los animales para realizar en laboratorio el despistaje de coccidia. Las raciones se prepararon semanalmente en forma manual, el LS se adicionó a la ración uniformizándolo previamente con un 10 por ciento del subproducto de trigo hasta obtener una mezcla homogénea, para luego agregarlo al total de la ración, la cual se depositó en sacos de polipropileno para su almacenamiento y protección del medio ambiente. El alimento se proporcionó a los corderos una vez al día, a las siete de la mañana, regulándose el suministro del alimento en función al consumo mostrado por los animales, agregándose un 10 por ciento más de alimento con respecto al consumo mostrado la semana anterior.

Tabla 2. Composición porcentual y costo promedio de las raciones tal como ofrecido por tratamiento.

\begin{tabular}{|c|c|c|c|}
\hline \multirow[b]{2}{*}{ Insumos* } & \multirow{2}{*}{$\begin{array}{r}\text { Costos } \\
(\mathrm{S} / \mathrm{kg})\end{array}$} & \multicolumn{2}{|c|}{ Tratamientos } \\
\hline & & $\begin{array}{l}\text { Testigo } \\
\text { (T1) \% }\end{array}$ & $\begin{array}{c}\text { Experimental } \\
\text { (T2) \% }\end{array}$ \\
\hline Maíz grano & 0,55 & 38,877 & 38,875 \\
\hline $\begin{array}{l}\text { Subproducto de } \\
\text { trigo }\end{array}$ & 0,25 & 22,142 & 22,141 \\
\hline Maíz panca & 0,15 & 13,094 & 13,093 \\
\hline $\begin{array}{l}\text { Pasta de algodón } \\
(36 \%)\end{array}$ & 0,43 & 12,000 & 11,999 \\
\hline $\begin{array}{l}\text { Harina de pescado } \\
(60 \%)\end{array}$ & 0,85 & 8,121 & 8,120 \\
\hline Melaza de caña & 0,30 & 3,000 & 2,999 \\
\hline $\begin{array}{l}\text { Carbonato de } \\
\text { calcio }\end{array}$ & 0,22 & 2,167 & 2,166 \\
\hline Sal común & 0,12 & 0,500 & 0,499 \\
\hline Zoodry VM-9P** & 7,40 & 0,100 & 0,099 \\
\hline $\begin{array}{l}\text { Lasalócido } \\
\text { sódico(\%15) }\end{array}$ & 21,65 & 0,000 & 0,004 \\
\hline Costos (S/kg) & & 0,43 & 0,431 \\
\hline
\end{tabular}

Los animales fueron pesados y medidos la talla, antes del suministro de alimento, semanalmente durante ocho semanas que duró el experimento. Las variables evaluadas y analizadas fueron: consumo de alimento, ganancia de peso vivo e incremento de talla, conversión alimenticia, eficiencia de utilización de los alimentos, valor económico, beneficio, calificación y rendimiento de carcasa.
Tabla 3. Valor nutritivo estimado en base a materia seca.

\begin{tabular}{lcc}
\hline \multirow{1}{*}{ Nutrientes } & \multicolumn{2}{c}{ Tratamientos } \\
& $\begin{array}{l}\text { Testigo } \\
\text { (T1) \% }\end{array}$ & $\begin{array}{c}\text { Experimental } \\
\text { (T2) \% }\end{array}$ \\
\hline Materia seca & 87,00 & 87,00 \\
Proteína cruda & 20,67 & 20,67 \\
Fibra cruda & 8,97 & 8,97 \\
Extracto etéreo & 3,83 & 3,83 \\
NDT $^{*}$ & 66,56 & 66,56 \\
\hline
\end{tabular}

* Según Bath et al. (1987).

$* \operatorname{NDT}(\%)=1,15(\%$ PC $)+1,75(\% \mathrm{EE})+0,45(\% \mathrm{FC})+$ $0,0085($ ELN2 \%) $+0,25($ ELN \% $)-3,4$.

Se utilizó el Análisis de Variancia Multivariado (MANOVA), que tradicionalmente se usa en estudios de datos con medidas repetidas en ensayos desarrollados en el sector agropecuario. Este modelo se utilizó para cada una de las variables que se evaluaron: ganancia de peso y talla, consumo de alimento y conversión alimenticia, siendo la covariable el peso inicial para todas las variables en estudio excepto para la talla cuya covariable fue la talla inicial. Para evaluar el rendimiento de carcasa (\%) se utilizó el Diseño Completamente al Azar con covariancia, siendo la covariable el peso inicial.

\section{RESULTADOS Y DISCUSIÓN}

El consumo de alimento acumulado, en base seca, fue de 55,81 kg/ovino y 56,49 kg/ovino para el T1 y T2, respectivamente, registrándose mayor consumo en el T2. Los datos sobre los incrementos de peso vivo se reportan en la Tabla 4, donde se aprecia que el consumo en ambos tratamientos se incrementa de acuerdo al transcurso de las semanas, tal como lo indica Maynard et al. (1981), quienes afirman que el consumo de materia seca de los animales en crecimiento se incrementa en relación a la edad del animal, debido a que los animales en crecimiento requieren cada vez, más nutrientes para cubrir sus diversas necesidades. Asimismo, Castellanos (1989), indica que el consumo de materia seca en ovinos de pelo está influenciado por el peso vivo del animal, así como por la energía metabolizable de la ración. 
Tabla 4. Ganancia de peso vivo individual y promedio por tratamiento.

\begin{tabular}{ccccc}
\hline Tratamiento & $\begin{array}{c}\text { Ovinos } \\
\mathbf{N}^{\circ} \text { Arete }\end{array}$ & $\begin{array}{c}\text { Peso } \\
\text { Inicial } \\
(\mathbf{k g})\end{array}$ & $\begin{array}{c}\text { Peso } \\
\text { final } \\
(\mathbf{k g})\end{array}$ & $\begin{array}{c}\text { Incremento } \\
\text { de peso* (kg) }\end{array}$ \\
\hline & 1 & 20,0 & 33,0 & 13 \\
Testigo & 3 & 21,5 & 33,5 & 12 \\
(T1) & 4 & 18,5 & 33,5 & 15 \\
& 24,0 & 36,2 & 12,25 \\
& 5 & 19,2 & 33,0 & 13,8 \\
\hline Promedio & & 20,64 & 33,8 & 13,21 \\
& & & 5 & \\
D. S.** & & 2,154 & 1,36 & 1,221 \\
\hline \multirow{2}{*}{ Experime } & 8 & 22,5 & 34,5 & 12,0 \\
ntal (T2) & 9 & 22,0 & 33,5 & 11,5 \\
& 10 & 19,5 & 34,0 & 14,5 \\
& 11 & 24,0 & 36,5 & 12,5 \\
\hline Promedio & & 21,667 & 34,7 & 13,083 \\
D. S.** & & 1,807 & 1,00 & 1,393 \\
\hline
\end{tabular}

*Incremento de peso vivo total (8 semanas).

***Desviación estándar.

Los valores ajustados de consumo acumulado son 63,84 $\mathrm{kg}$ y $63,97 \mathrm{~kg}$, respectivamente (Tabla 5). Estos resultados coinciden con los obtenidos por Foreyt et al. (1979) y Huston et al. (1990), quienes suplementando ovinos con lasalócido sódico, no encontraron diferencias significativas entre tratamientos. Una menor concentración de lasalócido sódico en la ración durante la primera semana no tiene un efecto en la reducción del consumo de alimento, similar a lo reportado por Horton y Stockdale (1981). Sin embargo, López (1992) encontró en el engorde intensivo de vacunos suplementados con lasalócido sódico, un mayor consumo de alimento diario promedio con respecto al control (Berger et al., 1981).

Al analizar los pesos vivos individuales (Tabla 4) y promedio por tratamiento al inicio y final del experimento; se observa que el incremento de peso vivo del T1 (13,21 kg), es ligeramente superior al T2 (13,08 $\mathrm{kg}$ ). Sin embargo, al realizar el análisis estadístico, los

lo cual pudo haber influenciado negativamente en los resultados. Además, diferencias en el concentrado, incrementos de peso totales ajustados no presentan diferencias significativas $(p>0,05)$ entre tratamientos (Tabla 5). Estos resultados coinciden con los reportados por Foreyt et al. (1981); Shetaewi y Ross (1987), y Thomas et al. (1990), quienes también emplearon diferentes dosis de lasalócido sódico en ovinos y en ganado vacuno (Jacques et al., 1987), que tampoco encontraron diferencias significativas en el incremento de peso. Los valores de incremento de peso fueron de 12,91 kg para el T1 y 13,33 kg para el T2 $(0,23 \mathrm{~kg} / \mathrm{día}$ y $0,24 \mathrm{~kg} / \mathrm{día}$ de incremento de peso vivo, respectivamente).

Tabla 5. Consumo de alimento promedio semanal por animal de las raciones experimentales por tratamiento $(\mathrm{kg})$.

\begin{tabular}{ccccc}
\hline \multirow{2}{*}{ Semana } & \multicolumn{2}{c}{ Testigo (T1) } & \multicolumn{2}{c}{$\begin{array}{c}\text { Experimental } \\
\text { (T2 con LS) }\end{array}$} \\
\cline { 2 - 5 } & $\begin{array}{c}\text { Tal como } \\
\text { ofrecido }\end{array}$ & $\begin{array}{c}\text { En base } \\
\text { seca }\end{array}$ & $\begin{array}{c}\text { Tal como } \\
\text { ofrecido }\end{array}$ & $\begin{array}{c}\text { En base } \\
\text { seca }\end{array}$ \\
\hline 1 & 6,08 & 5,344 & 6,293 & 5,531 \\
2 & 6,775 & 5,995 & 6,971 & 6,127 \\
3 & 7,245 & 6,368 & 7,383 & 6,49 \\
4 & 8,022 & 7,051 & 8,119 & 7,136 \\
5 & 8,8 & 7,735 & 8,717 & 7,662 \\
6 & 8,71 & 7,656 & 8,86 & 7,787 \\
7 & 8,87 & 7,796 & 9,004 & 7,914 \\
8 & 8,995 & 7,906 & 8,921 & 7,841 \\
\hline Total Periodo & 317,485 & 279,0 & 385,608 & 338,9 \\
Experimental & & 55 & & 28 \\
\hline Promedio/Ani & 63,497 & 55,81 & 64,268 & 56,48 \\
mal/Periodo & & 1 & & 8 \\
\hline Promedio/Ani & 1,058 & 0,930 & 1,071 & 0,941 \\
mal/día & & & & \\
\hline
\end{tabular}

La edad de los animales podría ser un factor que influye en el resultado, tal como se aprecia en el experimento utilizando corderos destetados precozmente (35 días) se evaluaron con diferentes niveles de monensina y lasalócido sódico por un período de 103 días, obteniéndose mejores resultados con dosis de 25 y 50 $\mathrm{mg}$ de LS por kg de alimento (Horton y Stockdale, 1981). En el presente estudio, la edad de los animales debido a la raza y exigencia de mercado fue de 5-6 meses para ser beneficiado a los 6-7 meses,

nivel de forraje y número de animales son factores que también podrían alterar los resultados de la prueba. 
Tabla 6. Consumo de alimento, ganancia de peso y conversión alimenticia.

\begin{tabular}{lcc}
\hline \multirow{2}{*}{\multicolumn{1}{c}{ Parámetros }} & \multicolumn{2}{c}{ Tratamientos } \\
\cline { 2 - 3 } & $\begin{array}{c}\text { Testigo } \\
\text { (T1) \% }\end{array}$ & $\begin{array}{c}\text { Experi } \\
\text { mental } \\
\text { (T2) \% }\end{array}$ \\
\hline Número de animales & 5 & 6 \\
Ganancia de peso total & $12,911^{\mathrm{a}}$ & $13,332^{\mathrm{a}}$ \\
Ganancia de peso total por día & 0,231 & 0,238 \\
Consumo de alimento total & $63,849^{\mathrm{a}}$ & $63,975^{\mathrm{a}}$ \\
(kg) & 1,14 & 1,142 \\
Consumo de alimento día (kg) & 36,974 & 37,045 \\
Total de NDT consumido (kg) & 34,92 & 35,99 \\
E.U.A (\%) & $4,945^{\mathrm{a}}$ & $4,799^{\mathrm{a}}$ \\
CA (Base fresca) & 4,302 & 4,175 \\
CA (Base seca) & & \\
\hline
\end{tabular}

${ }^{a}$ Letras similares indican que no hay diferencia estadística significativa $(\mathrm{p}>0,05)$.

En experimentos realizados con corderos én nacidos o destetados hubo un efecto significativo en la ganancia de peso (Horton y Stockdale, 1981; Foreyt et al., 1979;
Foreyt et al., 1981a); mientras que en ovinos de más edad no se encontró diferencias significativas (Huston et al., 1990), aunque si se reportó una mayor retención de nitrógeno (Ricke et al., 1984). Horton y Stockdale (1981), sostienen que las respuestas a la alimentación con lasalócido sódico, en corderos podrían deberse a sus efectos terapéuticos de este sobre la coccidiosis clínica, debido a que la coccidia puede reducir el consumo de alimento y la ganancia de peso.

Por otra parte, las tallas individuales de los ovinos al inicio y final del experimento, así como también los incrementos de tallas reales se presentan en la Tabla 7 , donde se aprecia en ambos tratamientos, un crecimiento regular de los ovinos durante toda la etapa experimental. Los incrementos de talla real promedio fueron de $10.3 \mathrm{~cm}$ para el tratamiento testigo (T1) y 10.0 $\mathrm{cm}$ para el tratamiento experimental (T2), no encontrándose diferencias estadísticas significativas entre tratamientos $(\mathrm{p}>0,05)$. Siendo los incrementos de talla finales ajustados para el T1 de $10,19 \mathrm{~cm}$ y para el T2 $10,09 \mathrm{~cm}$.

Tabla 7. Tallas individuales e incrementos de tallas reales

\begin{tabular}{|c|c|c|c|c|c|c|}
\hline \multirow{2}{*}{ Tratamiento } & \multirow{2}{*}{ Ovinos $\mathbf{N}^{\circ}$ Arete } & \multicolumn{2}{|c|}{ Control de Talla } & \multirow{2}{*}{$\begin{array}{c}\text { Incremento } \\
\text { total }(\mathbf{c m})\end{array}$} & \multirow{2}{*}{$\begin{array}{l}\text { Incremento de } \\
\text { talla total }\end{array}$} & \multirow{2}{*}{$\begin{array}{l}\text { Incremento de } \\
\text { talla por día }\end{array}$} \\
\hline & & Inicial (cm) & Final (cm) & & & \\
\hline \multirow{5}{*}{$\begin{array}{c}\text { Testigo } \\
\text { (T1) }\end{array}$} & 1 & 49,0 & 61,0 & 12,0 & \multirow{5}{*}{$10,09^{\mathrm{a}}$} & \multirow{5}{*}{0,182} \\
\hline & 2 & 53,5 & 63,0 & 9,5 & & \\
\hline & 3 & 55,0 & 63,0 & 8,0 & & \\
\hline & 4 & 52,0 & 63,5 & 11,5 & & \\
\hline & 5 & 51,5 & 62,0 & 10,5 & & \\
\hline Promedio & & 52,4 & 52,4 & 10,3 & & \\
\hline D.S.** & & 2,152 & 2,152 & 1,60 & & \\
\hline \multirow{6}{*}{$\begin{array}{l}\text { Experimen } \\
\text { tal (T2) }\end{array}$} & 6 & 55,5 & 62,0 & 6,5 & \multirow{6}{*}{$10,09^{\mathrm{a}}$} & \multirow{6}{*}{0,018} \\
\hline & 7 & 51,5 & 61,0 & 9,5 & & \\
\hline & 8 & 51,5 & 61,5 & 10,0 & & \\
\hline & 9 & 53,5 & 63,0 & 9,5 & & \\
\hline & 10 & 48,0 & 62,0 & 14,0 & & \\
\hline & 11 & 56,5 & 67,0 & 10,5 & & \\
\hline Promedio & & 52,75 & 52,75 & 10,0 & & \\
\hline D.S.** & & 3,094 & 2,19 & 2,41 & & \\
\hline
\end{tabular}

a Letras similares indican que no hay diferencia estadística significativa $(\mathrm{p}>0,05)$.

En animales que están en crecimiento existe una alta correlación positiva entre peso y talla; en tal sentido, debido a que no hubo diferencia estadística entre las tallas de ambos tratamientos tampoco hubo diferencias estadísticas entre los pesos de ambos tratamientos (Acuña, 1993). 
La conversión alimenticia (CA) y la eficiencia de la utilización de los alimentos (E.U.A.) obtenidos en el presente estudio para cada tratamiento, se muestran en la Tabla 6, donde se observa que no existe diferencias estadísticas significativas entre tratamientos $(\mathrm{p}>0,05)$. Los valores de conversión alimenticia acumulado de 4,94 para el T1 y 4,80 para el T2, lo que representa una mejora en 3,03 por ciento. Estos resultados fueron similares a los obtenidos por Ricke et al. (1984); Foreyt et al. (1981) y Foreyt et al. (1986). Asimismo, Jacques et al. (1987), en ganado de carne, tampoco encontró mejoras significativas en la conversión alimenticia.

Los resultados del valor económico de engorde de los tratamientos testigo y experimental, se presentan en la Tabla 8. Al analizar los tres factores que determinan la eficiencia económica, se observa que el factor consumo de alimento y la ganancia de peso total favorecen ligeramente a la ración experimental. Sin embargo, el precio de la ración es el factor determinante de la ración más eficiente. Se observa en la Tabla 8, que la ración experimental (T2), es más eficiente que la ración testigo (T1), necesitándose menos soles (2,12 vs. 2,06), para ganar un kilo de peso vivo por animal.

Tabla 8. Valor económico por tratamiento.

\begin{tabular}{lcc}
\hline \multirow{1}{*}{ Parámetros } & \multicolumn{2}{c}{ Tratamientos } \\
\cline { 2 - 3 } & $\begin{array}{l}\text { Testigo } \\
\text { (T1) \% }\end{array}$ & $\begin{array}{c}\text { Experimental } \\
\text { (T2) \% }\end{array}$ \\
\hline $\begin{array}{l}\text { Total de alimento tal } \\
\text { como ofrecido (kg)* } \\
\text { Ganancia de peso total } \\
\text { (kg)* }\end{array}$ & 63,849 & 63,975 \\
$\begin{array}{l}\text { Conversión } \\
\text { alimenticia }\end{array}$ & 12,911 & 13,332 \\
$\begin{array}{l}\text { Costo de ración } \\
\text { (Soles/kg) }\end{array}$ & 4,945 & 4,779 \\
& 0,43 & 0,431 \\
$\begin{array}{l}\text { Soles necesarios para } \\
\text { ganar un kg de Peso }\end{array}$ & 4,945 & 4,799 \\
Vivo & 4,302 & 4,175 \\
\hline
\end{tabular}

El rendimiento promedio de los animales al beneficio y los rendimientos de carcasa promedio se reportan en la Tabla 9. Siendo los rendimientos promedio reales de 48,67\% para el T1 y 46,60\% para el T2. Al realizar el análisis estadístico de los rendimientos de carcasas se observó que no existen diferencias estadísticas significativas entre tratamientos $(\mathrm{p}>0,05)$. Siendo los rendimientos de carcasa ajustados de 48,60 \% para el T1 y 46,66 \% para el T2.

Estos resultados coinciden con los reportados por Owens et al. (1982) y Nelson y Landblom (1983) aun cuando estos autores no determinaron diferencias significativas entre tratamientos para el parámetro rendimiento de carcasa.

Tabla 9. Rendimiento promedio para ambos tratamientos.

\begin{tabular}{lcc}
\hline \multirow{1}{*}{ Parámetros } & \multicolumn{2}{c}{ Tratamientos } \\
\cline { 2 - 3 } & $\begin{array}{c}\text { Testigo } \\
\text { (T1) \% }\end{array}$ & $\begin{array}{c}\text { Experimental } \\
\text { (T2) \% }\end{array}$ \\
\hline $\begin{array}{l}\mathrm{N}^{\circ} \text { de animales } \\
\text { Peso vivo promedio } \\
(\mathrm{kg})\end{array}$ & 52,05 & 6 \\
$\begin{array}{l}\text { Peso de carcasa } \\
\text { promedio (kg) }\end{array}$ & 15,60 & 15,61 \\
$\begin{array}{l}\text { Rendimiento promedio } \\
\text { (\%) }\end{array}$ & 48,674 & 46,597 \\
$\begin{array}{l}\text { Rendimiento promedio } \\
\text { ajustado (\%) }\end{array}$ & $48,599^{\mathrm{a}}$ & $46,658^{\mathrm{a}}$ \\
$\begin{array}{l}\text { Número total de } \\
\text { carcasas }\end{array}$ & 5 & 6 \\
$\begin{array}{l}\text { Número de carcasas } \\
\text { extra }\end{array}$ & 5 & 6 \\
Porcentaje extra & 100 & 100 \\
\hline
\end{tabular}

En cuanto al análisis de los resultados sobre la clasificación de las carcasas (Tabla 9), no existieron diferencias entre tratamientos. Todas las carcasas de ambos tratamientos se clasificaron como extras, por cumplir con los requisitos necesarios en cuanto a buena conformación de masa muscular y buena distribución de la misma; buen acabado. Asimismo, la grasa de cobertura apropiada con una buena distribución sobre los músculos superficiales de la paleta, dorso y costillares, así como también grasa de infiltración adecuada (MINAG, 1995). 


\section{CONCLUSIONES}

Las variables consumo de alimento, ganancia de peso, conversión alimenticia, incremento de talla y rendimiento de carcasa no fueron influenciados significativamente por la adición del lasalócido sódico en raciones de ovinos de pelo de la raza Blackbelly en crecimiento. En el análisis del valor económico, la ración del tratamiento experimental fue más eficiente que la ración del tratamiento testigo; necesitándose menos soles (S/ 2,0126 vs. S/ $2,068)$ para ganar un kilo de peso vivo. Se recomienda determinar el efecto de la adición del lasalócido sódico en el alimento de inicio en corderos Blackbelly y efectuar comparaciones del uso del lasalócido sódico con otros ionóforos en raciones de engorde en ovinos.

\section{Conflictos de intereses}

Los autores firmantes del presente trabajo de investigación declaran no tener ningún potencial conflicto de interés personal o económico con otras personas $\mathrm{u}$ organizaciones que puedan influir indebidamente con el presente manuscrito.

\section{Contribuciones de los autores}

Preparación y ejecución: JAG, BCV, JCS; Desarrollo de la metodología: JAG, BCV, JCS; Concepción y diseño: JAG, BCV, JCS; Edición del artículo: JAG, BCV, JCS; Supervisión del estudio: JAG, BCV, JCS.

\section{LITERATURA CITADA}

- Acuña, R. (1993). Uso del lasalócido sódico como promotor del crecimiento en la alimentación de terneras Holstein .Tesis Ingeniero Zootecnista. Universidad Nacional Agraria La Molina, Lima. Perú. 101 p.

- Bartley, E., Herod, E., Bechtle, R., Sapienza, D., Brent, B. \& Davidovich, A. (1979). Effect of monensin or lasalocid, with and without niacin or amicloral, on rumen fermentation and feed efficiency. Journal of Animal Science, 49(4), 1066-1075.

- Bergen, W. \& Bates, D. (1984). Ionophores: their effect on production efficiency and mode of action. Journal of animal science, 58(6), 14651483.

- Berger, L., Ricke, S. \& Fahey Jr, G. (1981). Comparison of two forms and two levels of lasalocid with monensin on feedlot cattle performance. Journal of animal science, 53(6), 1440-1445.

- Carro, M., Ranilla, M. \& Tejido, M. 2006. Utilización de aditivos en la alimentación del ganado ovino y caprino. Pequeños Rumiantes, 7(3), 26-37.

- Castellanos, A. (1989). Requerimientos alimenticios del borrego pelibuey. En tecnología para la producción de ovejas tropicales. Publicación FAO, Santiago de Chile. 165p.

- Chen, M. \& Wolin, M. (1979). Effect of monensin and lasalocid-sodium on the growth of methanogenic and rumen saccharolytic bacteria. Appl. Environ. Microbiol, 38(1), 72-77.

- Dennis, S., Nagaraja, T. \& Bartley, E. (1981). Effect of lasalocid or monensin on lactateproducing or using rumen bacteria. Journal of Animal Science, 52(2), 418-426.

- Foreyt, W., Gates, N. \& Rich, J. $\left(1981^{\mathrm{a}}\right)$. Evaluation of lasalocid in salt against ovine Coccidia. American journal of veterinary research, 42(1), 54-56.

- Foreyt, W., Parish, S. \& Foreyt, K. (1981b). Lasalocid for improved weight gains and control of Coccidia in lambs. American journal of veterinary research, 42(1), 57-60.

- Foreyt, W., Rice, D. \& Wescott, R. (1986). Evaluation of lasalocid as a coccidiostat in calves: titration, efficacy, and comparison with monensin and decoquinate. American Journal of Veterinary Research, 47(9), 2031-2035.

- Fuller, J. \& Johnson, D. (1981). Monensin and lasalocid effects on fermentation in vitro. Journal of Animal Science, 53(6), 1574-1580.

- Horton, G. \& Stockdale, P. (1981). Lasalocid and monensin in finishing diets for early weaned lambs with naturally occurring coccidiosis. American journal of veterinary research, 42(3), 433-436.

- Huston, J., Engdahl, B. \& Calhoun, M. (1990). Effects of supplemental feed with or without ionophores on lambs and Angora kid goats on rangeland. Journal of animal science, 68(12), 3980-3986. 
- Jacques, K., Cochran, R., Corah, L., Avery, T., Zoelliner, K. \& Higginbotham, J. (1987). Influence of lasalocid level on forage intake, digestibility, ruminal fermentation, liquid flow and performance of beef cattle grazing winter range. Journal of animal science, 65(3), 777-785.

- Jorquera, A. \& Angulo, A. (2016). Evaluación de aditivos alimenticios sobre la respuesta productiva de ovinos finalizados en corral. $1^{\circ}$ Seminario Internacional Ovino-Caprino, La Habana, Cuba.

- Kirk, D. \& Fontenot, J. (1987). Effect of lasalocid and monensin upon site of absorption and digestive tract flow of minerals in sheep. J. Auim. Sci, 65 (1), 455.

- López, A. (1992). Efecto de la suplementación con lasalócido sódico en el engorde intensivo de vacunos .Tesis Ingeniero Zootecnista. Universidad Nacional Agraria La Molina, Lima. Perú. 109 p.

- Maynard, D., Loosli, J., Hintz, H., Warner, R. (1981). Nutrición Animal. Editorial Mc GrewHill. México.

- Michelis, D. \& Sánchez, D. 2016. Uso de monensina en dietas para caprinos. Tesis Doctoral, Universidad Nacional de La Plata, La Plata, Argentina. 27p.

- MiNAG [MINISTERIO DE AGRICULTURA]. (1995). Reglamento Tecnológico de Carnes Decreto Supremo N 22-95-AG. Lima, Perú.

- Shetaewi, M. \& Ross, T. (1987). Effect of supplementation with concentrates and lasalocid during late pregnancy and lactation on productivity of Rambouillet ewes and development of wool follicles in their lambs. Journal of animal science, 65(2):351-358.

- Nelson, J. \& Landblom, D. (1983). A comparison of rumensin and bovatec fed in wintering rations to beef cattle. 33rd Livestock Research Round-Up, Dickinson expt. Sta., Dickinson, North Dakota.

- Owens, F. \& Gill, D. (1982). Lasalocid for feedlot steers. Animal Science Res. Report MP: 112. Agric. Expt. Sta. Oklahoma State University and USDA-SEA-AR. 134p

- Paienter, G., Pollack, R. \& Pressman, B. (1982). Conformacional dynamics of the carboxylic ionophore lasolocid. An underlying cation complexation - decomplexation and membrane transport. Biochemistry, 21,5613.

- Paterson, J., Anderson, B., Bowman, D., Morrison, R. \& Williams, J. (1983). Effect of protein source and lasalocid on nitrogen digestibility and growth by ruminants. Journal of animal science, 57(6), 1537-1544.

- Pordomingo, A., Galyean, M., Branine, M. \& Freeman, A. (1999). Effects of daily and weekly rotations of lasalocid and monensin plus tylosin compared with continuous feeding of each ionophore on daily dry matter intake and digesta kinetics. Rev Argent Prod Anim, 19: 383-90.

- Pressman, B. \& Fahim, N. (1982). Pharmacology and toxicology of the monovalent carboxylic ionophores. Annual review of pharmacology and toxicology, 22(1),465-490.

- Ricke, L., Berger, L., Van Der Aar, P. \& Fahey, G. 1984. Effects of lasalocid and monensin on nutrient, digestion, metabolism and rumen characteristics of sheep. J. Anim. Sci., 58:194.

- Roche. (1986). Bovatec (lasalocid sódico). Boletín de servicio e información técnica. Lima. Perú16 .p.

- Roche. (1988). Productos para la nutrición y sanidad animal. Lima - Perú 2da. Edición.

- Thomas, V. (1989). Bovatec Improves Reproductive Rate. Montana Farmer Stockman. 49 Ref.

- Thomas, V., McInerney, M., Ayers, E. \& Kott, R. (1990). Influence of lasalocid and supplement level on productivity of gestating ewes grazing winter range. Journal of animal science, 68(6), 1530-1535.

- Valdivia, A., Matos, M., Rodríguez, Z., Pérez, Y., Rubio, Y. \& Vega, J. (2019). Enzymatic additives and their use on animal rearing. Cuban Journal of Agricultural Science, 53(4), 341-352. 